\section{Metastatic Crohn's disease of the umbilicus}

Non-contiguous disease of the skin in Crohn's disease (termed metastatic Crohn's disease ${ }^{1}$ ) is an unusual manifestation. We report on a patient with anorectal Crohn's and separate metastatic disease of the umbilicus.

\section{Case report}

A 46-year-old Caucasian woman presented with a six-month history of severe perianal discomfort and pain on defecation, associated with bleeding, alternating diarrhoea and constipation, and weight loss. Coincidentally with her symptoms she had noticed a sore at the umbilicus.

Examination showed a painful, swollen, moist, inflamed ubilicus. Perianal examination showed multiple fissures, two low-level posterior anal fistulae, and several large oedematous purple skin tags. Sigmoidoscopy disclosed friable, oedematous mucosa with ulceration. Rectal biopsy specimens showed severe chronic inflammatory changes confined mainly but not entirely to the mucosa, but no granulomata. A biopsy specimen of the umbilical lesion showed chronic inflammatory changes with several noncaseating granulomata and giant cells, but no acid-fast bacilli (figure).

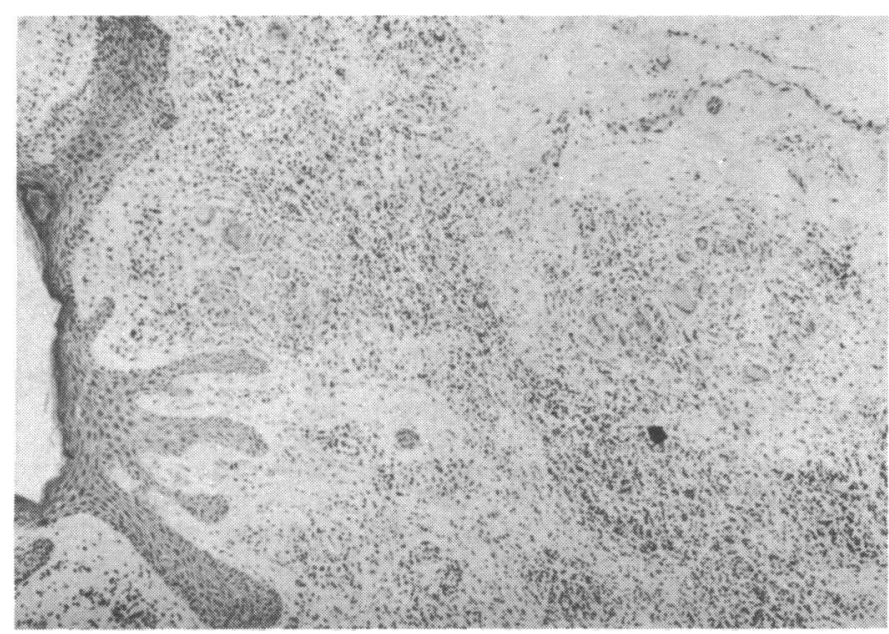

Typical "sarcoid" reaction in umbilical biopsy specimen showing noncaseating granulomata, Langhan's giant cells, and epithelioid cells. $\times 50$ (original magnification).

Other investigations showed mild hypochromic anaemia (haemoglobin concentration $10.3 \mathrm{~g} / \mathrm{dl})$, raised erythrocyte sedimentation rate $(82 \mathrm{~mm}$ in first hour), normal white blood cell count $\left(6.4 \times 10^{9}\right)$, slight hypokalaemia (serum potassium concentration $3.0 \mathrm{mmol}(\mathrm{mEq}) / 1$, and hypoalbuminaemia (serum albumin concentration $29 \mathrm{~g} / \mathrm{l}$ ). Liver function tests, and the blood concentrations of urea, other electrolytes, and trace elements were normal Sputum and urine cultures failed to show any acid-fast bacilli, and Mantoux test result was negative. Chest radiography showed no evidence of sarcoidosis or tuberculosis, and barium meal and follow-through showed no abnormality. A barium enema, performed with difficulty, was normal, apart from ulceration visible to the lower sigmoid region.

Topical steroid treatment could not be tolerated and in view of the severe rectal pain on defecation she was treated with total parenteral nutrition and nil by mouth for 33 days, together with prednisolone $10 \mathrm{mg}$ thrice daily. She responded well to treatment with haemoglobin concentration rising to $11.7 \mathrm{~g} / \mathrm{dl}$ and erythrocyte sedimentation rate falling to $32 \mathrm{~mm}$ in first hour. Umbilical and perianal lesions also improved and she became pain free and able to defecate. One year later she remains in remission on a maintenance oral dose of prednisolone $2.5 \mathrm{mg}$ thrice daily.

\section{Comment}

Patients with skin disease from Crohn's disease fall into two groups. One group comprises several different dermatoses ${ }^{2}$ (pyoderma gangrenosum, erythema nodosum, erythema multiforme, palmar erythema, rosacea, epidermolysis bullosa acquisita, and polyarteritis nodosa) with varying histology. The other group have a "sarcoid" reaction as described by Parks et al, typically epithelioid cells and Langhan's-type giant cells in a non-caseating granuloma, together with a chronic inflammatory infiltrate. There have been several reports of such lesions, most of which spread outwards in a contiguous fashion from the perineum towards the scrotum and penis ${ }^{34}$ or from a diseased stoma ${ }^{5}$; oral disease by similar lesions has been noted, but the mouth might be considered as part of the alimentary tract.

True non-contiguous metastatic skin disease is extremely rare, usually occurring where there is skin apposition within flexures such as the sub-mammary, ${ }^{1}$ retroauricular, ${ }^{2}$ or anterior abdominal-wall skin fold ${ }^{4}$ regions. Metastatic Crohn's disease of the umbilicus has not been reported, but this area is similarly one of skin apposition.

It is not clear whether metastatic Crohn's disease may precede alimentary tract disease, as may perianal lesions, but in our patient it developed simultaneously with the rectal disease, and both responded in parallel to treatment. It is important, nevertheless, to consider Crohn's disease in patients presenting with sarcoid-like skin lesions, even in the absence of overt alimentary symptoms or signs. As the histology is non-specific, tuberculosis and sarcoidosis must also be excluded. The cause of metastatic Crohn's disease remains an enigma.

1 Parks AG, Morson BC, Pegum JS. Crohn's disease with cutaneous involvement. Proc $R$ Soc Med 1964;58:241-2.

${ }^{2}$ McCallum DI, Gray WM. Metastatic Crohn's disease. Br $\mathcal{F}$ Dermatol $1976 ; 95: 551-4$

${ }^{3}$ Cockburn AG, Krolikowski J, Balogh K, Roth RA. Crohn's disease of penile and scrotal skin. Urology 1980;15:596-8.

4 Mountain JC. Cutaneous ulceration in Crohn's disease. Gut 1970;11: 18-26.

5 Taylor VE, Smith CJ. Oral manifestations of Crohn's disease without demonstrable gastro-intestinal lesions. Oral Surg 1975;39:58-66.

(Accepted 1 fuly 1981)

St Mary's Hospital, London W9

R K S PHILLIPS, FRCS, surgical registrar

GEOFFREY GLAZER, FRCS, consultant surgeon

\section{Burns caused by striking underground electricity cables}

Effective preventative measures are the most important factor in dealing with the problem of burns. Industrial accidents are still a regular and preventable cause. When such accidents entail damage to underground electricity cables the result may be immediate death or severe injuries, including burns. A few of those injured in this way are referred to burns units and a review of patients admitted to our unit shows the type of avoidable injury which these accidents can cause.

\section{Case reports}

From June 1979 to December 1980, eight previously fit men aged 16-55 years were admitted for treatment of burns caused by striking underground cables. Six had damaged the cables with pneumatic drills and two with forks. Three, with $9 \%, 12 \%$, and $18 \%$ burns affecting the face and hands were inpatients for 12 to 28 days and their burns healed with regular dressings. One with $23 \%$ superficial burns required resuscitation with intravenous fluids but healed with regular dressings as an inpatient for eight days. Three men, with $25 \%, 25 \%$, and $40 \%$ burns were inpatients for 37 to 61 days; they needed resuscitation with intravenous fluids and two operations each for débridement of the wounds and skin grafting, in addition to regular dressings. One patient in this group had an inhalation injury, requiring temporary endotracheal intubation and ventilation, and another had a pulmonary embolus after operation. One 30-year-old man had a $44 \%$ burn and despite prompt adequate resuscitation developed a severe haemolytic anaemia with acute renal failure, and died three days after his accident. All patients had flash burns, with or without additional flame burns from burning clothes. The man who died also had signs of electrical, as opposed to heat, burns on his hands. ${ }^{1}$

\section{Comment}

Each year there are about 20000 incidents due to underground electricity cables in Britain. ${ }^{2}$ Although only a few of these accidents 